\title{
Optimization of evaporative cooling
}

\author{
C. A. Sackett, C. C. Bradley, and R. G. Hulet \\ Physics Department and Rice Quantum Institute, Rice University, Houston, Texas 77005-1892
}

(Received 29 July 1996)

\begin{abstract}
Recent experiments have used forced evaporative cooling to produce Bose-Einstein condensation in dilute gases. The evaporative cooling process can be optimized to provide the maximum phase-space density with a specified number of atoms remaining. We show that this global optimization is approximately achieved by locally optimizing the cooling efficiency at each instant. We discuss how this method can be implemented, and present the results for our ${ }^{7} \mathrm{Li}$ trap. The predicted behavior of the gas is found to agree well with experiment. [S1050-2947(97)07005-4]
\end{abstract}

PACS number(s): 32.80.Pj, 42.50.Vk, 51.10.+y, 44.60.+k

The recent observations of Bose-Einstein condensation (BEC) in dilute atomic vapors have opened an exciting new area of physics research [1-3]. These experiments have relied on forced evaporative cooling (FEC) to produce gases with high phase-space density $\rho$. In this process, the hottest atoms are selectively removed from a system and the remainder are allowed to rethermalize via elastic two-body collisions, resulting in a colder and more dense distribution. A comprehensive review is given in Ref. [4].

The technique is implemented by confining spin-polarized atoms in a magnetic trap with potential energy $U(\mathbf{r})$. Atoms are removed by driving transitions between trapped and untrapped spin states using an oscillating radio-frequency field. The untrapped atom is expelled from the trap by a potential energy $U^{*}(\mathbf{r})$. Because the energy difference $U(\mathbf{r})-U^{*}(\mathbf{r})$ has a strong monotonic spatial dependence, an applied field oscillating with angular frequency $\Omega$ will be resonant only at positions $\mathbf{r}$ such that $U(\mathbf{r})-U^{*}(\mathbf{r})=\hbar \Omega$. Atoms with total energy $E<U(\mathbf{r})$ will not be found at $\mathbf{r}$, and thus will remain trapped, while atoms with greater energies will be lost. In this way, the frequency $\Omega$ defines the depth of the trap, $E_{t}$. Elastic collisions among the remaining colder atoms replenish the high-energy tail of the distribution, allowing evaporation to continue. As this proceeds, $E_{t}$ is lowered to keep pace with the decreasing energy of the atoms.

During evaporation, the trapped atoms are not in thermal equilibrium, but $\rho$ can be defined to be the phase-space density that would result if the gas were allowed to equilibrate. If, during a time $d t$, evaporation changes the number of trapped atoms $N$ by $d N$ while $\rho$ changes by $d \rho$, the efficiency of evaporation is defined to be

$$
\gamma=-\frac{d \rho / \rho}{d N / N}
$$

In a lossless trap, in which atoms are removed only by evaporation, the efficiency can be made arbitrarily large by setting $E_{t}$ to a large value. However, in real traps there are loss mechanisms that continually act on the atoms, and if $E_{t}$ is made too large, the slow increase in $\rho$ due to evaporation will be overwhelmed by the decrease due to losses. FEC therefore depends on both the elastic collision rate $R$, which determines the rate of rethermalization, and the trap loss rate $\Gamma$. In general, the larger the ratio $R / \Gamma$, the more efficient evaporation can be.

Given a system with specified $R$ and $\Gamma$, it is desirable to know where best to place $E_{t}$. More precisely, for a given initial temperature and number of trapped atoms, we wish to determine the function $E_{t}(t)$ that maximizes the phase-space density when a final number $N_{f}$ of trapped atoms is reached.

Several models of evaporative cooling have been proposed [5-13]. The task is simplified by the fact that, for bosons at low temperatures, collisions have purely $s$-wave character, and that during evaporation the motion of the trapped atoms is classical. Even with these simplifications, however, modeling the detailed evolution of the gas is computationally intensive. Simple Monte Carlo techniques are prohibitively slow, although more sophisticated Monte Carlo methods have recently been demonstrated that may overcome this obstacle $[12,13]$. The most precise technique is to treat the trapped gas as a continuous fluid obeying the Boltzmann transport equation [14]. A model of this sort was developed by Luiten et al. [11], and is used here. They develop the model for a general trapping potential, but the discussion here is specific to the most practical case of a harmonic oscillator potential $U(\mathbf{r})=m \omega^{2} r^{2} / 2$.

At time $t$, the trapped atoms are characterized by a distribution function $f(\mathbf{r}, \mathbf{p}, t)$, normalized so that

$$
d N=\frac{d^{3} r d^{3} p f(\mathbf{r}, \mathbf{p})}{(2 \pi \hbar)^{3}}
$$

is the number of atoms at position $\mathbf{r}$ with momentum $\mathbf{p}$. The evolution of $f$ is governed by the Boltzmann equation [14]

$$
\left(\frac{\mathbf{p}}{m} \cdot \nabla_{\mathbf{r}}-\left(\nabla_{\mathbf{r}} U\right) \cdot\left(\nabla_{\mathbf{p}}\right)+\frac{\partial}{\partial t}\right) f(\mathbf{r}, \mathbf{p})=I(\mathbf{r}, \mathbf{p})-\Gamma(\mathbf{r}) f(\mathbf{r}, \mathbf{p}) .
$$

The right-hand side describes the effects of collisions. The elastic term $I$ is given by 


$$
\begin{aligned}
I\left(\mathbf{r}, \mathbf{p}_{1}\right)= & \frac{\sigma}{4 \pi^{4} m \hbar^{3}} \int d^{3} p_{2} d^{3} p_{3} d^{3} p_{4} \delta^{3}\left(\mathbf{p}_{1}+\mathbf{p}_{2}-\mathbf{p}_{3}-\mathbf{p}_{4}\right) \\
& \times \delta\left(p_{1}^{2}+p_{2}^{2}-p_{3}^{2}-p_{4}^{2}\right)\left[f\left(\mathbf{r}, \mathbf{p}_{3}\right) f\left(\mathbf{r}, \mathbf{p}_{4}\right)\right. \\
& \left.-f\left(\mathbf{r}, \mathbf{p}_{1}\right) f\left(\mathbf{r}, \mathbf{p}_{2}\right)\right]
\end{aligned}
$$

where $\sigma$ is the $s$-wave elastic cross section. The loss rate $\Gamma$ comprises both the rate for trapped atoms to collide with hot background gas molecules and the rate for inelastic collisions between trapped atoms:

$$
\Gamma(\mathbf{r})=\Gamma_{1}+\frac{G_{2}}{(2 \pi \hbar)^{3}} \int d^{3} p f(\mathbf{r}, \mathbf{p}) .
$$

Here $G_{2}$ is the two-body inelastic rate constant. At high enough densities three-body or more collisions will also contribute, and Eq. (5) may easily be extended to account for them.

A convenient approximation to Eq. (3) is made by assuming that the motion of atoms in the trap is ergodic. The distribution function $f(\mathbf{r}, \mathbf{p})$ is then a function only of the energy $H(\mathbf{r}, \mathbf{p})=p^{2} /(2 m)+U(\mathbf{r})$, which clearly simplifies the equation of motion. Justification for this approximation is discussed in Refs. [11] and [15]. To test the approximation, we compared the results of our model to those of a direct Monte Carlo simulation of the atomic motion. The two models agreed, within the statistical fluctuations of the Monte Carlo results. Similar observations were made by Holland et al. [13]. However, Wu and Foot [12] point out that the ergodic approximation breaks down in the special case in which atoms can only be removed from a spatially restricted region of the constant-energy surface $U(\mathbf{r})=E_{t}$. The fast Monte Carlo technique they present is capable of handling such circumstances.

In the ergodic approximation, the energy distribution function $f(E)$ is defined such that the number of atoms with energy $E$ is

$$
d N=g(E) f(E) d E,
$$

where $g(E)$ is the density of states, given by

$$
g(E)=\frac{1}{(2 \pi \hbar)^{3}} \int d^{3} r d^{3} p \delta(E-H(\mathbf{r}, \mathbf{p}))=\frac{E^{2}}{2(\hbar \omega)^{3}}
$$

for a harmonic oscillator potential. The distribution functions $f(E)$ and $f(\mathbf{r}, \mathbf{p})$ are related by

$$
f(\mathbf{r}, \mathbf{p})=\int d E \delta(H(\mathbf{r}, \mathbf{p})-E) f(E),
$$

and $f(E)$ obeys

$$
g(E) \frac{\partial f(E)}{\partial t}=I(E)-\Gamma(E) g(E) f(E) .
$$

The collision integral $I$, as derived in Ref. [11], is

$$
\begin{aligned}
I\left(E_{1}\right)= & \frac{m \sigma}{\pi^{2} \hbar^{3}} \int d E_{2} d E_{3} d E_{4} g\left(E_{\min }\right) \delta\left(E_{1}+E_{2}-E_{3}-E_{4}\right) \\
& \times\left[f\left(E_{3}\right) f\left(E_{4}\right)-f\left(E_{1}\right) f\left(E_{2}\right)\right],
\end{aligned}
$$

where $E_{\min }=\min \left\{E_{1}, E_{2}, E_{3}, E_{4}\right\}$. The loss rate $\Gamma$ is

$$
\Gamma(E)=\Gamma_{1}+\frac{4(2 m)^{3 / 2}}{(\pi \hbar)^{3}} G_{2} \int_{0}^{\infty} d E^{\prime} \frac{E_{<}^{5 / 2}}{E^{2}} h\left(\frac{E_{>}}{E_{<}}\right) f\left(E^{\prime}\right),
$$

where $E_{<}$is the lesser of $\left\{E, E^{\prime}\right\}$ and $E_{>}$the greater. The function $h(x)$ is the definite integral

$$
h(x)=\int_{0}^{1} d z z^{2} \sqrt{1-z^{2}} \sqrt{x-z^{2}} .
$$

Evaporation is included by setting $f(E)=\partial f(E) / \partial t=0$ for $E>E_{t}$. Equations (9)-(11) can be generalized to the anisotropic harmonic oscillator by replacing $\omega$ with $\left(\omega_{x} \omega_{y} \omega_{z}\right)^{1 / 3}$ in $g(E)$. Calculation of an evaporation trajectory using Eq. (9) requires a few hours on a computer workstation.

The optimization problem is to determine the best trajectory from $N_{i}$ to $N_{f}$. Therefore, the optimization process requires variation of the entire trajectory $E_{t}(t)$ and calculation of the response of the atom distribution. It has been suggested, however, that the optimum $E_{t}$ can be found at each time $t$ using only the instantaneous response of the distribution, by choosing $E_{t}(t)$ to maximize the efficiency at time $t[5,4]$. This would simplify the calculation since the instantaneous response is given by Eq. (9) directly, while finding the response to the entire trajectory requires the solution of Eq. (9).

In a harmonic trap, $\rho \propto N /\langle E\rangle^{3}=N^{4} / E_{\text {tot }}^{3}$, where $\langle E\rangle$ is the average energy of the trapped atoms and $E_{\text {tot }}=N\langle E\rangle$ is the total trap energy. The efficiency $\gamma$ can therefore be expressed as

$$
\gamma=-\frac{d(\ln \rho)}{d(\ln N)}=3 \frac{N}{E_{\mathrm{tot}}} \frac{d E_{\mathrm{tot}}}{d N}-4
$$

The energy $E_{\text {tot }}=\int d E E g(E) f(E)$, and its derivative is expressed as

$$
\frac{d E_{\mathrm{tot}}}{d N}=\frac{d E_{\mathrm{tot}} / d t}{d N / d t}=\frac{\int d E E g(E) d f / d t}{\int d E g(E) d f / d t} .
$$

Equation (9) gives the derivative $d f / d t$, so the function $\gamma\left(E_{t}\right)$ is well defined and a single-variable optimization algorithm may be used to determine $E_{t}$. Rather than finding $E_{t}$ directly, it is more efficient to optimize the parameter $\eta=E_{t} /\langle E\rangle$, because the optimum value of $\eta$ changes more slowly as evaporation proceeds and requires less frequent optimization checks. It is not clear whether this method could be applied to a model in which the distribution function is determined by a Monte Carlo technique, since the derivatives in Eq. (14) would not be readily calculable.

This optimization method would be exact if the evolution of the system depended only on $N$ and $\rho$. This can be understood by considering the phase-space density produced by the instantaneously optimized trajectory, $\rho_{\text {inst }}(N)$, and that of an alternative trajectory $\rho_{\text {alt }}(N)$. Since $\rho_{\text {inst }}$ is optimized at the beginning of the trajectory, initially $\rho_{\text {alt }}<\rho_{\text {inst }}$. If 


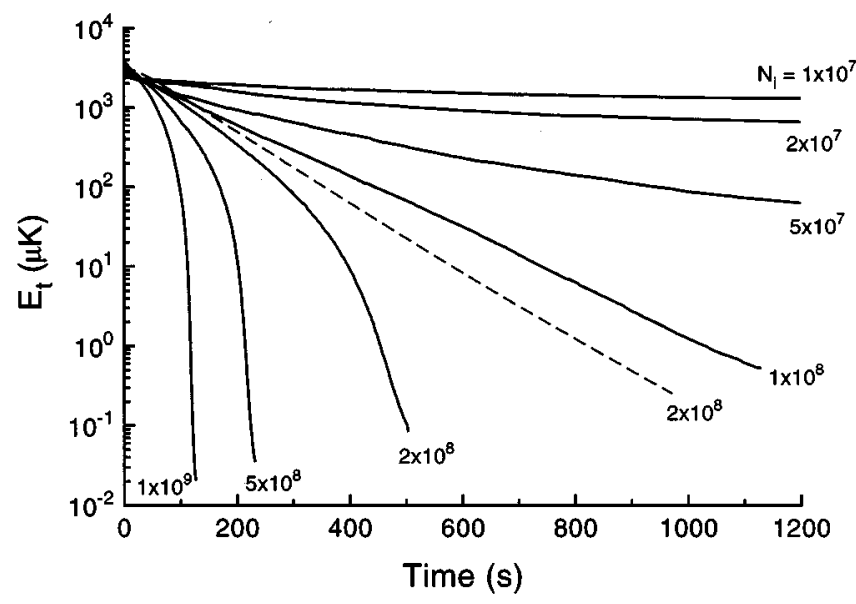

FIG. 1. Solid lines are optimized trajectories $E_{t}(t)$ for our ${ }^{7} \mathrm{Li}$ trap [16]. The initial number of trapped atoms is given by the label on each curve. The initial temperature is $500 \mu \mathrm{K}$ in each case. The trap oscillation frequency $\omega=\left(\omega_{x} \omega_{y} \omega_{z}\right)^{1 / 3}=2 \pi \times 135.5 \mathrm{~Hz}$, the one-particle loss-rate $\Gamma_{1}=(300 \mathrm{~s})^{-1}$, the two-body rate constant $G_{2}=1 \times 10^{-14} \mathrm{~cm}^{3} / \mathrm{s}$ [17], and the elastic cross section $\sigma=5 \times 10^{-13} \mathrm{~cm}^{2}[18]$. The response of the gas to these trajectories is given in Fig. 2. The dashed line is a nonoptimized trajectory, which gives the dashed curve shown in Fig. 2. While the trajectories for initial numbers less than $1 \times 10^{8}$ are only shown for times up to $1200 \mathrm{~s}$, the corresponding curves in Fig. 2 represent longer evaporation times.

$\rho_{\text {alt }}\left(N_{f}\right)>\rho_{\text {inst }}\left(N_{f}\right)$, the two trajectories must cross at some point $N^{\prime}$. This can only occur if

$$
-\frac{d \rho_{\mathrm{alt}}\left(N^{\prime}\right)}{d N}>-\frac{d \rho_{\mathrm{inst}}\left(N^{\prime}\right)}{d N},
$$

which is impossible by the construction of $\rho_{\text {inst }}(N)$. So, $\rho_{\text {inst }}(N)$ represents the optimum trajectory.

Because the evolution of the distribution does depend on the form of $f(E)$, this optimization scheme is only approximately correct. For instance, at $N^{\prime}, \rho_{\text {alt }}=\rho_{\text {inst }}$, but if $f_{\text {alt }}(E) \neq f_{\text {inst }}(E)$, then it is possible for Eq. (15) to hold, so that $\rho_{\text {alt }}\left(N_{f}\right)$ can be larger than $\rho_{\text {inst }}\left(N_{f}\right)$. By varying the shape of the distribution, we determined that a distribution starting with a larger high-energy tail can be driven more efficiently than one with a smaller high-energy tail, as might be expected. In principle, this fact can be used to construct a trajectory that is more efficient than $\rho_{\text {inst }}(t)$. However, it is unlikely that significant gain in phase-space density could be obtained in this way, because the efficiency possible at a given $N$ depends much more on $\rho$ than on the details of $f$. To test this sensitivity, we modeled evaporation in our trap starting from several nonequilibrium distributions with the same $N$ and $\rho$. We found that varying the initial number of atoms with $E>2\langle E\rangle$ by a factor of 3 produces the same variation in the final $\rho$ as is caused by a $5 \%$ variation of the initial total number.

We have determined the optimum trajectories in our ${ }^{7} \mathrm{Li}$ trap for a number of initial conditions. The values of $E_{t}$ versus time are shown in Fig. 1, and the corresponding paths of the trapped gas through phase space are given by the solid curves in Fig. 2. Although the assumption that the distribu-

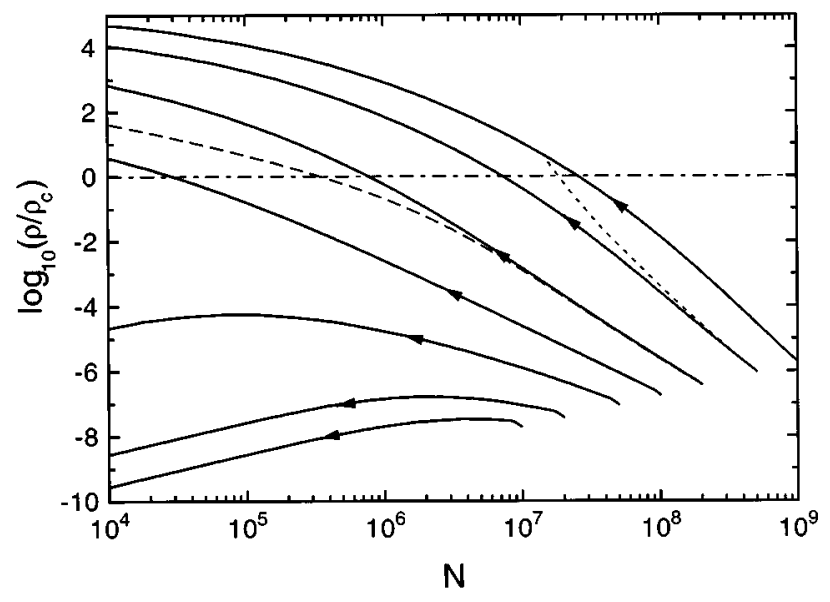

FIG. 2. Optimized trajectories through phase space. The arrows give the direction of time. The solid curves and the long-dashed curve represent the response of the trapped ${ }^{7} \mathrm{Li}$ gas to the trajectories shown in Fig. 1. The short-dashed curve shows the optimized results obtained in the absence of two-body losses, exhibiting runaway evaporation. The dot-dashed line shows the BEC transition, at $\rho=\rho_{c}=1.202 /(2 \pi \hbar)^{3}$. Note that the model used is only valid in the nondegenerate regime, but the trajectories are continued past the BEC transition to illustrate how a classical gas would behave.

tion function evolves classically breaks down sometime before the system crosses the BEC phase transition, the results of the model are shown past this point to illustrate the predicted behavior of a classical gas.

If the elastic collision rate and loss rate were constant, $E_{t}(t)$ would have an exponential form. As Fig. 1 shows, the optimum $E_{t}(t)$ is generally nonexponential. The varying curvatures of the trajectories come about because the collision rate and the loss rate vary differently with the number and temperature of the trapped atoms. To determine the benefit obtained by optimization, we compare with the results obtained using an exponential $E_{t}(t)$. The time constant of the exponential was determined using the method of Ref. [4] for a trajectory with $E_{t}=2\langle E\rangle$. The long-dashed curve in Fig. 1 shows the time dependence, and Fig. 2 shows the results of the calculation. The optimized trajectory reaches the BEC transition in $60 \%$ less time and with a factor of 2.4 larger $N$ than the nonoptimized trajectory. Although these gains are significant, the fact that the exponential trajectory performs as well as it does indicates the robustness of the evaporative cooling technique.

In addition to providing a greater $\rho\left(N_{f}\right)$, a set of optimized trajectories such as those shown in Fig. 2 also shows what initial conditions are required in order to obtain BEC with a specified final number of atoms. This allows for definite design goals when developing experiments, and provides a more detailed criterion than the previously understood requirement that the collision rate to loss rate ratio $R / \Gamma$ must be greater than about 150 [9].

Runaway evaporation is not observed for our conditions. This phenomenon is predicted to occur when $R / \Gamma$ increases as evaporation proceeds, which can happen if the density increases [4]. Once begun, this process would seem to produce arbitrarily large phase-space density increases in very little time. It would be manifested as an upward-curving $\rho$ versus $N$ trajectory. An example is the short-dashed curve in 


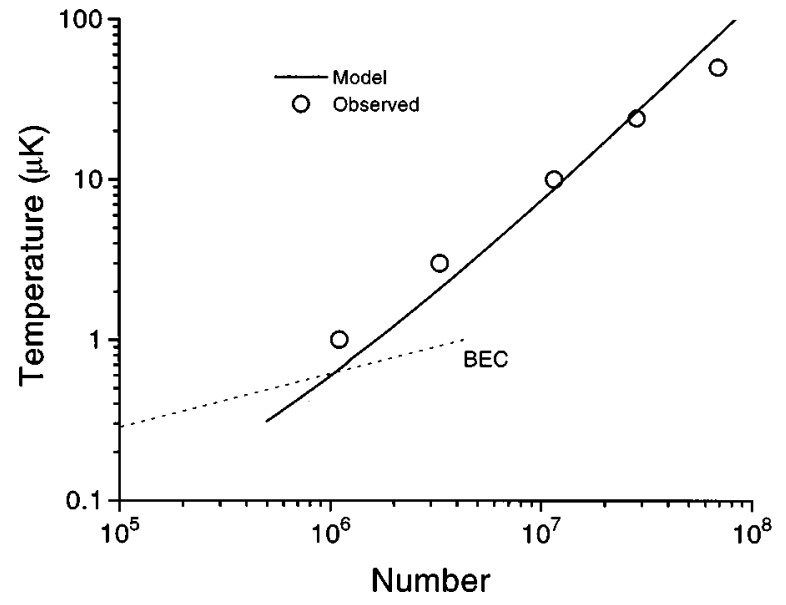

FIG. 3. Comparison of model and experiment. For each data point shown, the trap was loaded and then evaporatively cooled using the model trajectory shown. Evaporation was halted after a variable time, and the number and temperature of the remaining atoms were measured. The discrepancies observed are within the uncertainties of the measurement, starting conditions, and trap loss rates.

Fig. 2, which is an optimized trajectory calculated with no two-body losses. A few of the real trajectories shown in the figure do have slight upward curvature at their outsets, but the effect is small and all eventually level out and approach zero efficiency. This is due to the quenching effect of inelastic two-body collisions. The elastic collision rate $R$ is approximately $n \sigma v$, while the loss rate $\Gamma \approx \Gamma_{1}+G_{2} n$. So, the ratio $R / \Gamma$ approaches $\sigma v / G_{2}$ for large $n$, which decreases as $T$ is lowered. Runaway evaporation therefore ceases when $G_{2} \approx \Gamma_{1}$, or $n \approx 3 \times 10^{11} \mathrm{~cm}^{-3}$ for our experiment.

We compare the predictions of the evaporative cooling model with experimental results in Fig. 3. The experimental procedures and apparatus have been described in previous publications $[2,16]$. The data points were obtained by loading the trap, evaporatively cooling using a set $\Omega(t)$ for specified lengths of time, and then taking a picture of the atom distribution using a brief flash of laser light and a chargecoupled device camera. The colder points on the graph were obtained by imaging the absorption of an axial probe beam, while warmer points were obtained by imaging the fluorescence of the atoms induced by a balanced set of transverse beams. The agreement between experiment and model is good, and the discrepancies observed are probably due to uncertainties in the measurements, the initial conditions and the trap loss rates. Further investigations should provide interesting tests of the ergodic approximation as well as the breakdown of the model as BEC is approached.

Determination of optimized trajectories using the method described is computationally slow. The calculation can be simplified by approximating the distribution at all times by a truncated Boltzmann distribution, which allows the integrals in Eq. (9) to be calculated analytically [11]. Luitten et al. tested this approximation using a cooling trajectory in which $E_{t}$ was held constant and $N$ allowed to fall by a factor of 2 . Although they found good agreement with the exact result, this is an inadequate test for the experimental situation in which $N$ decreases by many orders of magnitude. We tested the effect of the approximation using our optimization model, and observed that it resulted in a small overestimate of the efficiency of cooling. When cooling from $N_{i} \sim 10^{8}$ to $N_{f}=10^{5}$, using the approximation gave the same $\rho\left(N_{f}\right)$ as obtained by increasing $N_{i}$ by $5 \%$ to $10 \%$. For sufficiently high $N_{i}$, the effect on $\rho\left(N_{f}\right)$ is of the same order of magnitude, and the approximation is probably adequate. As Fig. 2 indicates, however, if $N_{i}$ is too low FEC does not work. Near the threshold for success, the behavior of the model is very sensitive to the initial conditions, and the Boltzmann approximation is not valid.

In conclusion, we have presented a technique for determining the optimum trajectory for forced evaporative cooling. In most cases the Boltzmann approximation can be used, allowing fast computation. The model predicts that optimization can yield significant increases in phase-space density, but it also shows that FEC is fairly robust to variations of the trajectory and initial conditions, if the initial conditions are sufficiently favorable. This robustness makes FEC a very powerful experimental technique.

This work was supported by the National Science Foundation, the Welch Foundation, and the Texas Advanced Technologies Program. C.A.S. acknowledges the support of the NSF.
[1] M. J. Anderson, J. R. Ensher, M. R. Matthews, C. E. Wieman, and E. A. Cornell, Science 269, 198 (1995).

[2] C. C. Bradley, C. A. Sackett, J. J. Tollett, and R. G. Hulet, Phys. Rev. Lett. 75, 1687 (1995).

[3] K. B. Davis, M.-O. Mewes, M. R. Andrews, N. J. van Druten, D. S. Durfee, D. M. Kurn, and W. Ketterle, Phys. Rev. Lett. 75, 3969 (1995).

[4] W. Ketterle and N. J. van Druten, in Advances in Atomic, Molecular and Optical Physics, edited by B. Bederson and $\mathrm{H}$. Walther (Academic Press, San Diego, 1996), No. 36.

[5] H. F. Hess, Phys. Rev. B 34, 3476 (1986).

[6] T. Tommila, Europhys. Lett 2, 789 (1986).

[7] D. W. Snoke and J. P. Wolfe, Phys. Rev. B 39, 4030 (1989).
[8] J. M. Doyle, J. C. Sandberg, I. A. Yu, C. L. Cesar, D. Kleppner, and T. J. Greytak, Physica B 194-196, 13 (1994).

[9] C. R. Monroe, E. A. Cornell, C. A. Sackett, C. J. Myatt, and C. E. Wieman, Phys. Rev. Lett. 70, 414 (1993).

[10] K. B. Davis, M.-O. Mewes, and W. Ketterle, Appl. Phys. B 60, 155 (1995).

[11] O. J. Luiten, M. W. Reynolds, and J. T. M. Walraven, Phys. Rev. A 53, 381 (1996).

[12] H. Wu and C. J. Foot, J. Phys. B 29, 321 (1996).

[13] M. Holland, J. Williams, K. Coakley, and J. Cooper, Quantum Semiclass. Opt. 8, 571 (1996).

[14] K. Huang, Statistical Mechanics, 2nd ed. (John Wiley and Sons, New York, 1987). 
[15] E. L. Surkov, J. T. M. Walraven, and G. V. Shlyapnikov, Phys. Rev. A 53, 3403 (1996).

[16] J. J. Tollett, C. C. Bradley, C. A. Sackett, and R. G. Hulet, Phys. Rev. A 51, R22 (1995).
[17] A. J. Moerdijk and B. J. Verhaar, Phys. Rev. A 53, R19 (1996).

[18] E. R. I. Abraham, W. I. McAlexander, C. A. Sackett, and R. G. Hulet, Phys. Rev. Lett. 74, 1315 (1995). 